\title{
An Edge-Signed Generalization of Chordal Graphs, Free Multiplicities on Braid Arrangements, and Their Characterizations
}

\author{
Takuro $\mathrm{Abe}^{1}$, Koji Nuida $^{2}$ and Yasuhide Numata ${ }^{34}$ \\ ${ }^{1}$ Department of Mathematics, Kyoto University, Kitashirakawa-Oiwake-cho, Sakyo-ku, Kyoto 606-8502, Japan \\ ${ }^{2}$ Research Center for Information Security (RCIS), National Institute of Advanced Industrial Science and Technology \\ (AIST), Akihabara-Daibiru Room 1003, 1-18-13 Sotokanda, Chiyoda-ku, Tokyo 101-0021, Japan \\ ${ }^{3}$ Department of Mathematical Informatics, The University of Tokyo, 7-3-1 Hongo, Bunkyo-ku, Tokyo 113-0033, \\ Japan \\ ${ }^{4}$ Japan Science and Technology Agency (JST), CREST, Sanbancho, Chiyoda-ku, Tokyo, 102-0075, Japan
}

\begin{abstract}
In this article, we propose a generalization of the notion of chordal graphs to signed graphs, which is based on the existence of a perfect elimination ordering for a chordal graph. We give a special kind of filtrations of the generalized chordal graphs, and show a characterization of those graphs. Moreover, we also describe a relation between signed graphs and a certain class of multiarrangements of hyperplanes, and show a characterization of free multiarrangements in that class in terms of the generalized chordal graphs, which generalizes a well-known result by Stanley on free hyperplane arrangements. Finally, we give a remark on a relation of our results with a recent conjecture by Athanasiadis on freeness characterization for another class of hyperplane arrangements.

Résumé. Dans cet article, nous proposons une généralisation de la notion des graphes triangulés à graphes signés, qui est basé sur l'existence d'un ordre d'élimination simplicial à un graphe triangulé. Nous donnons un genre spécial de filtrations des graphes triangulés généralisés, et montrons une caractérisation de ces graphes. De plus, nous décrivons aussi une relation entre graphes signés et une certaine classe de multicompositions d'hyperplans, et montrons une caractérisation de multicompositions libres dans cette classe en termes des graphes triangulés généralisés, qui généralise un résultat célèbre de Stanley sur compositions libres d'hyperplans. Finalement, nous donnons une remarque sur une relation de nos résultats avec une conjecture récente d'Athanasiadis sur une caractérisation du freeness d'une autre classe de compositions d'hyperplans.
\end{abstract}

Keywords: hyperplane arrangement, free arrangement, chordal graph, signed graph, characterization

\section{Introduction}

Let $V^{\ell}$ be an $\ell$-dimensional vector space over a field $\mathbb{K}$ of characteristic zero. A hyperplane arrangement $\mathcal{A}$ (or simply an arrangement) is a finite collection of affine hyperplanes in $V^{\ell}$. In this article any arrangement $\mathcal{A}$ is assumed, unless otherwise specified, to be central, i.e., each hyperplane in $\mathcal{A}$ contains 
the origin. A multiplicity on an arrangement $\mathcal{A}$ is a map $m: \mathcal{A} \rightarrow \mathbb{Z}_{\geq 0}$, and a pair $(\mathcal{A}, m)$ is called a $m u l$ tiarrangement. Then an arrangement is a multiarrangement $(\mathcal{A}, m)$ with $m$ constantly equal to one, that is also called a simple arrangement. Theory of (multi)arrangements is an intersecting area of geometry, algebra and combinatorics (see e.g., Orlik-Terao (1992)). For example, some associated combinatorial objects such as intersecting lattices and characteristic polynomials of an arrangement reflect properties of the arrangement. On the other hand, braid arrangements, or more generally Coxeter arrangements, are fundamental objects in the theory of arrangements that are closely related to root systems of finite Coxeter groups (see e.g., Saito (1975)).

One of the aims of this article is to give insight into freeness property of multiarrangements, that is one of the most active topics in theory of (multi)arrangements, in a combinatorial viewpoint specified below. To define the freeness property, we need some definitions and notations. Let $\left\{x_{1}, \ldots, x_{\ell}\right\}$ denote a basis for the dual vector space $V^{*}=\left(V^{\ell}\right)^{*}$ of $V^{\ell}$, and let $S=\operatorname{Sym}\left(V^{*}\right) \simeq \mathbb{K}\left[x_{1}, \ldots, x_{\ell}\right]$ be the symmetric algebra on $V^{*}$. Let $\operatorname{Der}_{\mathbb{K}}(S)=\bigoplus_{i=1}^{\ell} S \cdot \partial_{x_{i}}$ denote the $S$-module of $\mathbb{K}$-linear derivations of $S$. For each hyperplane $H \in \mathcal{A}$ in a given multiarrangement $(\mathcal{A}, m)$, fix a linear form $\alpha_{H} \in V^{*}$ such that $\operatorname{ker}\left(\alpha_{H}\right)=H$. Then $(\mathcal{A}, m)$ is called free if the logarithmic derivation module $D(\mathcal{A}, m)$ of $(\mathcal{A}, m)$ defined by

$$
D(\mathcal{A}, m)=\left\{\theta \in \operatorname{Der}_{\mathbb{K}}(S) \mid \theta\left(\alpha_{H}\right) \in S \cdot \alpha_{H}^{m(H)} \text { for all } H \in \mathcal{A}\right\}
$$

is a free $S$-module (of rank $\ell$ ). Moreover, since for any free $(\mathcal{A}, m)$ the $S$-module $D(\mathcal{A}, m)$ has a free basis $\left\{\theta_{1}, \ldots, \theta_{\ell}\right\}$ such that each $\theta_{i}$ is homogeneous of degree $\operatorname{deg}\left(\theta_{i}\right)$ (i.e., $\theta_{i}=\sum_{j=1}^{\ell} f_{i, j} \partial_{x_{j}}$ with every $f_{i, j}$ either zero or homogeneous of degree $\operatorname{deg}\left(\theta_{i}\right)$ ), and in that case the multiset of the degrees $\operatorname{deg}\left(\theta_{i}\right)$ is independent of the choice of the basis, we define the exponents of a free multiarrangement $(\mathcal{A}, m)$ by

$$
\exp (\mathcal{A}, m)=\left\{\operatorname{deg}\left(\theta_{1}\right), \ldots, \operatorname{deg}\left(\theta_{\ell}\right)\right\} \quad \text { (as a multiset) }
$$

When an arrangement $\mathcal{A}$ is fixed, we say that a multiplicity $m$ on $\mathcal{A}$ is free if $(\mathcal{A}, m)$ is a free multiarrangement. It is very difficult to determine free (multi)arrangements in general, and derivation modules and free (multi)arrangements in some special cases have been well studied in preceding works. For instance, see Abe-Terao-Wakefield (2008, 2007); Saito (1975, 1980); Solomon-Terao (1998); Terao (2002).

In this article, we deal with multiplicities on a braid arrangement $\mathcal{A}_{\ell}$ (equivalently, the Coxeter arrangement of type $A_{\ell}$ ) that is an arrangement in $V^{\ell+1}$ defined by

$$
\mathcal{A}_{\ell}=\left\{H_{i j}=\left\{x_{i}-x_{j}=0\right\} \mid i, j \in\{1,2, \ldots, \ell+1\}, i \neq j\right\} .
$$

More generally, a Coxeter arrangement is the arrangement of all reflecting hyperplanes of a finite Coxeter group. There have been several preceding results on free multiplicities on braid arrangements and Coxeter arrangements (see e.g., Abe (2007, preprint 2008); Abe-Yoshinaga (preprint 2007); Saito (1975, 1980); Solomon-Terao (1998); Terao (2002); Yoshinaga (preprint 2007)). In particular, a characterization of free multiplicities $m$ on Coxeter arrangements of the form $m(H)=c+\delta_{H}$, with $c$ a constant and $\delta_{H} \in\{0,1\}$ for every $H \in \mathcal{A}$, has been obtained by combining results of Abe-Yoshinaga (preprint 2007); SolomonTerao (1998); Terao (2002); Yoshinaga (2002). In this article, we consider any multiplicity $m$ on braid arrangements of another form $m(H)=2 k+\delta_{H}$, with $k \in \mathbb{Z}_{>0}$ a constant and $\delta_{H} \in\{-1,0,1\}$ for every $H \in \mathcal{A}$. Motivations of focusing on such situations are explained in the next paragraph. We parameterize such a multiplicity $m$ by using a signed graph with $\ell+1$ vertices in the following manner (see $e . g$., Diestel (2006) for graph-theoretic terminology): For a signed graph $G=(V, E)$ with $V=\left\{v_{1}, \ldots, v_{\ell+1}\right\}$ and 
$E=E_{+} \cup E_{-}$(disjoint union), define an auxiliary map $m_{G}: \mathcal{A}_{\ell} \rightarrow\{-1,0,1\}$ by

$$
m_{G}\left(H_{i j}\right)= \begin{cases}1 & \text { if } v_{i} v_{j} \in E_{+} \\ -1 & \text { if } v_{i} v_{j} \in E_{-} \\ 0 & \text { otherwise }\end{cases}
$$

where $v_{i} v_{j}$ denotes an unordered pair of $v_{i}$ and $v_{j}$, and put $m=2 k+m_{G}$. (In this article, every graph is finite, simple, and undirected, unless otherwise specified.) One of the main theorems in this article gives a characterization of free multiplicities on $\mathcal{A}_{\ell}$ of the above type in terms of a certain property of the corresponding signed graph that will be described below. More precisely, our result shows that, under a certain technical and not essential condition, a multiplicity $m=2 k+m_{G}$ is free if and only if $G$ is signed-eliminable in the sense specified in Section 2 . See Theorem 2 for the precise statement of the result. The notion of signed-eliminable graphs is a generalization of chordal graphs to signed graphs, and the above result is also a signed-graphic generalization of Stanley's well-known result (Stanley (1972)) on a characterization of free graphic arrangements in terms of chordal graphs. Moreover, Theorem 2 also shows that, if a multiplicity $m=2 k+m_{G}$ is free, then its exponents are determined by certain quantities associated to the signed graph $G$. A main ingredient of the proof of the result is a characterization of signed-eliminable graphs in terms of excluded subgraphs, that is another main contribution of this article. See Theorem 1 for the precise statement.

The main motivations of studying multiplicities of the above form $m(H)=2 k+\delta_{H}, \delta_{H} \in\{-1,0,1\}$, on a braid arrangement are as follows. First, it is known that a kind of duality exists between multiplicities $2 k+\delta_{H}$ and $2 k-\delta_{H}$, with all $\delta_{H} \in\{0,1\}$, not only for braid arrangements but also for Coxeter arrangements of other types (Abe (preprint 2008)). The authors had guessed that such a duality would extend to more general cases $\delta_{H} \in\{-1,0,1\}$; the work in this article on the case of braid arrangements is the first step to a study of the case of general Coxeter arrangements. Secondly, from the viewpoint of Stanley's freeness characterization based on (non-signed) chordal graphs, it is reasonable to expect that extending non-signed graphs to signed graphs gives a natural generalization of Stanley's theory, and the corresponding multiplicities are actually of the above type. Moreover, it will be mentioned in Section 5 that our study on the above multiplicities is closely related to a conjecture by Athanasiadis (Athanasiadis (2000) ) on freeness characterization for another class of arrangements.

This article is organized as follows. In Section 2 we give a definition of signed-eliminable graphs and introduce some related objects. We also show two inductive properties of signed-eliminable graphs that play key roles of the proof of our main theorems. Moreover, we present some quantities associated to signed-eliminable graphs that are main ingredients of the description of exponents of free multiarrangements of the above type. In Section 3 , we state one of the two main theorems of this article that characterizes signed-eliminable graphs in terms of excluded subgraphs (Theorem 1p. We also show an outline of the proof and some key lemmas that would be of independent interest. Moreover, we present some simpler characterizations of signed-eliminable graphs in certain subclasses as easy consequences of our result. In Section 4, we state an aforementioned result on free multiplicities on braid arrangements, that is another main theorem of this article (Theorem 2), and show an outline of the proof. As an application, we also describe characteristic polynomials of free multiarrangements of the above type (Corollary 57. Finally, in Section 5, we give a remark on a relation of our result with a conjecture by Athanasiadis (Athanasiadis (2000)) on freeness characterization for another class of arrangements. More precisely, our result is applied to prove one direction of Athanasiadis's Conjecture (the sufficiency of Athanasiadis's 
conditions for the freeness) in a more general setting than that in the statement of the conjecture.

\section{Signed-Eliminable Graphs}

First, we give a definition of signed-eliminable graphs:

Definition 1 For a signed graph $G=(V, E)$ and a bijection $\nu: V \rightarrow\{1,2, \ldots,|V|\}$ (in this article, such a map $\nu$ is referred to as an ordering on $G$ ), we say that $\nu$ is a signed-elimination ordering (or an SEO in short) if for any triple $(u, v, w)$ of vertices of $G$ such that $\nu(u)<\nu(w)>\nu(v)$, and for each $\sigma \in\{+,-\}$, the following two conditions are satisfied:

(E1) If $u w \in E_{\sigma}$ and $v w \in E_{\sigma}$, then $u v \in E_{\sigma}$.

(E2) If $u v \in E_{\sigma}$ and $v w \in E_{-\sigma}$, then $u w \in E_{\sigma}$.

We say that $G$ is signed-eliminable (or $\mathrm{SE}$ in short) if an SEO on $G$ exists.

See Figure 1 for the conditions (E1) and (E2), where single and duplicated edges represent edges with different signs. Note that, owing to a well-known characterization of chordal graphs in terms of vertex elimination orderings (see e.g., Fulkerson-Gross (1965)), both subgraphs $G_{+}=\left(V, E_{+}\right)$and $G_{-}=$ $\left(V, E_{-}\right)$of an SE graph are chordal. In particular, SE graphs with either $E_{+}=\emptyset$ or $E_{-}=\emptyset$ are nothing but chordal graphs. Two examples of SE graphs are given in Figure 2 An SEO for the graph in the left-hand side is given by $w \mapsto 1$ and $v_{i} \mapsto i+1$. On the other hand, for the graph in the right-hand side, an SEO is given by $w_{i} \mapsto i$ and $v_{i} \mapsto i+2$.
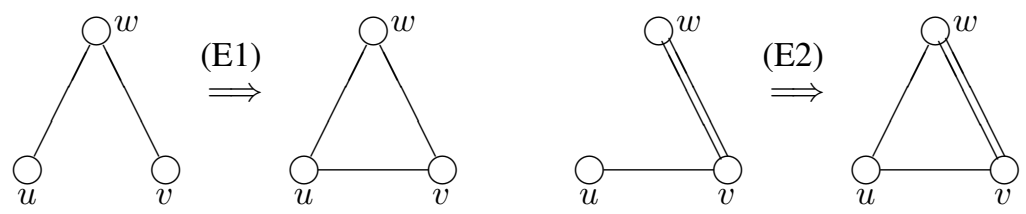

Fig. 1: Condition for signed-eliminable graphs
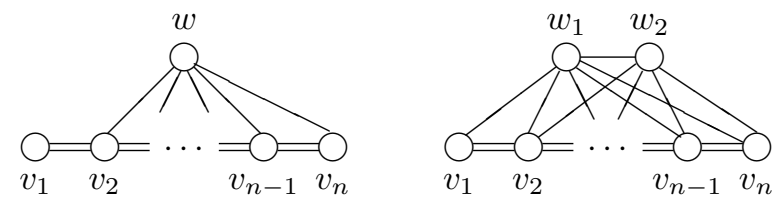

Fig. 2: Examples of SE graphs

Remark 1 The SEOs are also characterized in the following manner: We assign weights $\omega(u v)$ to pairs of vertices $u, v$ of a signed graph $G$ by the rule $\omega(u v)= \pm 1$ and 0 if $u v \in E_{ \pm}$and $u v \notin E$, respectively. Then an ordering $\nu$ on $G$ is an SEO if and only if, for any triple $(u, v, w)$ with $\nu(u)<\nu(w)>\nu(v)$ and either $u w \in E$ or $v w \in E$, if $a \leq b \leq c$ are three weights $\omega(u v), \omega(v w)$, and $\omega(u w)$ in nondecreasing order, then we have $b=\omega(u v)$. This property plays a key role in our characterization of free multiplicities of the above type. 
We summarize some fundamental properties of SE graphs. For any SEO $\nu$ on an SE graph $G$, the restriction of $\nu$ to an induced subgraph of $G$ gives an SEO on that subgraph. Hence the class of SE graphs is closed under taking induced subgraphs. On the other hand, a signed graph is SE if and only if every connected component of the graph is SE. Moreover, SE graphs have the following inductive properties. To explain the properties, we introduce the following terminology:

Definition 2 Let $G$ be a signed graph. Then a vertex $v$ of $G$ is called signed-simplicial if the following two conditions are satisfied:

(S1) For each $\sigma \in\{+,-\}, N_{G_{\sigma}}[v]=\left\{u \in V \mid u v \in E_{\sigma}\right\} \cup\{v\}$ is a clique in $G_{\sigma}$ (i.e., $v$ is a simplicial vertex of the graph $G_{\sigma}$ ).

(S2) For each $\sigma \in\{+,-\}$, $u w \in E_{-\sigma}$ and $w v \in E_{\sigma}$ imply $u v \in E_{-\sigma}$.

Moreover, let $S(G)$ denote the set of the signed-simplicial vertices of $G$.

Then we have the following result:

Lemma 1 If $G$ is an SE graph with an SEO $\nu$, then the vertex $v$ of $G$ with $\nu(v)=|V|$ is signed-simplicial in $G$ (and by the aforementioned property, the restriction of $\nu$ to the induced subgraph $G \backslash\{v\}$ of $G$ with vertex set $V \backslash\{v\}$ is an SEO on the subgraph). Conversely, if $G$ is a signed graph, $v \in S(G)$ and $\nu$ is an SEO on $G \backslash\{v\}$, then the unique extension $\bar{\nu}$ of $\nu$ to $G$ with $\bar{\nu}(v)=|V|$ is also an SEO on $G$.

This inductive property plays a central role in the proof of our characterization of SE graphs. Moreover, Lemma 1 implies that an SEO of any SE graph is found by a greedy algorithm, namely:

Lemma 2 We consider the following inductive algorithm for a signed graph $G$ : If $S(G)$ is empty then halt, otherwise let $v_{n}$ be a vertex in $S(G)$, where $n=|V|$, and proceed the algorithm for $G \backslash\left\{v_{n}\right\}$. Then $G$ is an $S E$ graph if and only if the algorithm does not halt until the graph becomes empty, and the ordering $\nu$ on $G$ with $\nu\left(v_{i}\right)=i$ determined in this way is an SEO if $G$ is an SE graph.

In contrast to the above inductive property with respect to vertices, the next property of SE graphs is inductive with respect to edges. We introduce the following notion:

Definition 3 Let $G$ be an $S E$ graph with an $S E O \nu$. For each $0 \leq k \leq|V|$, let $G^{(k)}$ denote the subgraph of $G$ with the same vertex set $V$ and an edge set consisting of all edges uv of $G$ such that $\nu(u) \leq k$ and $\nu(v) \leq k$. Then we say that a sequence $G_{0}^{\prime}=G^{(k-1)}, G_{1}^{\prime}, \ldots, G_{r}^{\prime}=G^{(k)}$ of subgraphs of $G$ is a $k$-th signed-eliminable filtration (or a $k$-th SE filtration in short) of $G$ if each $G_{i}^{\prime}(1 \leq i \leq r$ ) is obtained from $G_{i-1}^{\prime}$ by adding one edge and $\nu$ is also an SEO on $G_{i}^{\prime}$. Moreover, we refer to a concatenation of $k$-th $S E$ filtrations for all $1 \leq k \leq|V|$ as a complete signed-eliminable filtration (or a complete SE filtration in short) of $G$.

Then we have the following property, that plays a significant role in our characterization of free multiplicities of the above type:

Proposition 1 Any SE graph has a complete SE filtration.

We give an outline of construction of complete SE filtrations. For an SE graph $G$ with an SEO $\nu$, let $v \in V$ with $\nu(v)=|V|$. Then we define a binary relation $\prec$ on $N_{G}(v)=N_{G}[v] \backslash\{v\}$ by $u \prec w$ if and only if $u w \in E_{\sigma}, u v \in E_{\sigma}$ and $w v \in E_{-\sigma}$ for some $\sigma \in\{+,-\}$. It is shown that the transitive closure $\prec^{\prime}$ of $\prec$ is a partial order on $N_{G}(v)$, and that for a maximal element $u$ of $N_{G}(v)$ with respect to $\prec^{\prime}$, the 
subgraph of $G$ obtained by deleting the edge $u v$ is also an SE graph with the same $\operatorname{SEO} \nu$. Thus repetition of this process gives a desired filtration of $G$.

For example, for the SE graph $G$ in the left-hand side of Figure 2 with the SEO $\nu$ specified above, the unique $(n+1)$-th SE filtration of $G$ is given by first adding the edge $w v_{n}$ and then adding the edge $v_{n-1} v_{n}$, therefore a complete SE filtration is also inductively obtained. On the other hand, for the other graph $G$ in Figure 2 with the above SEO $\nu$, the unique $(n+2)$-th SE filtration of $G$ is given by adding three edges $w_{1} v_{n}, w_{2} v_{n}$, and $v_{n-1} v_{n}$ in this order, therefore a complete SE filtration is also inductively obtained.

In the last of this section, we introduce the following quantities associated to any SE graph that will be used to describe the exponents of free multiplicities of the above type. Let $G$ be an SE with an SEO $\nu$. Then we define $d_{\sigma}^{(\nu)}(i) \in \mathbb{Z}_{\geq 0}$ for each $1 \leq i \leq|V|$ and $\sigma \in\{+,-\}$ by

$$
d_{\sigma}^{(\nu)}(i)=\mid\left\{u \in V \mid \nu(u) \leq i \text { and } u_{i} u \in E_{\sigma}\right\} \mid,
$$

where $u_{i} \in V$ such that $\nu\left(u_{i}\right)=i$. Moreover, for each $i$, put

$$
\widetilde{\operatorname{deg}_{i}}=\widetilde{\operatorname{deg}_{i}}(G)=d_{+}^{(\nu)}(i)-d_{-}^{(\nu)}(i) .
$$

For example, for an SE graph $G$ in Figure 3 and an SEO $\nu$ in the left-hand side of Figure 3 , we have $\left(d_{+}^{(\nu)}(i), d_{-}^{(\nu)}(i)\right)=(0,0),(1,0),(0,0)$, and $(1,1)$ for each $i=1, \ldots, 4$, respectively. On the other hand, for an SEO $\mu$ in the right-hand side of Figure 3 , we have $\left(d_{+}^{(\mu)}(i), d_{-}^{(\mu)}(i)\right)=(0,0),(0,0),(1,1)$, and $(1,0)$ for each $i=1, \ldots, 4$, respectively. Now we see that the multisets of the pairs $\left(d_{+}^{(\nu)}(i), d_{-}^{(\nu)}(i)\right)$ in the first case and of the pairs $\left(d_{+}^{(\mu)}(i), d_{-}^{(\mu)}(i)\right)$ in the second case coincide with each other. This phenomenon is not just an accident, namely we have the following property (that coincides with Theorem 4 of Rose (1970) in the special case of non-signed graphs):
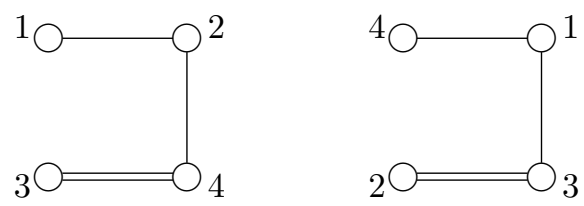

Fig. 3: Two SEOs on the same SE graph

Proposition 2 For any $S E$ graph $G$, the multiset of the pairs $\left(d_{+}^{(\nu)}(i), d_{-}^{(\nu)}(i)\right), 1 \leq i \leq|V|$, does not depend on the choice of an SEO $\nu$ on $G$. In particular, the multiset $\widetilde{\operatorname{deg}}(G)$ of the values $\widetilde{\operatorname{deg}}_{i}(G)$ is also independent on the choice of $\nu$.

Note that we always have $\widetilde{\operatorname{deg}_{1}}=0$ in the above setting.

\section{Characterization of Signed-Eliminable Graphs}

In this section, we give a characterization of SE graphs. To state the characterization, we need some more definitions: 
Definition 4 We say that a sequence $\left(v_{1}, v_{2}, \ldots, v_{n} ; w\right)$ of vertices with $n \geq 3$ is a $\sigma$-mountain, where $\sigma \in\{+,-\}$ (or simply a mountain), if $v_{i} v_{i+1} \in E_{-\sigma}$ for $1 \leq i \leq n-1$, wv $v_{i} \in E_{\sigma}$ for $2 \leq i \leq n-1$, and any other pair of vertices is not joined by an edge (see the left-hand side of Figure 4).

Definition 5 We say that a sequence $\left(v_{1}, v_{2}, \ldots, v_{n} ; w_{1}, w_{2}\right)$ of vertices with $n \geq 2$ is a $\sigma$-hill, where $\sigma \in\{+,-\}$ (or simply a hill), if $v_{i} v_{i+1} \in E_{-\sigma}$ for $1 \leq i \leq n-1, w_{1} w_{2} \in E_{\sigma}, w_{1} v_{i} \in E_{\sigma}$ for $1 \leq i \leq n-1, w_{2} v_{i} \in E_{\sigma}$ for $2 \leq i \leq n$, and any other pair of vertices is not joined by an edge (see the right-hand side of Figure 4 .
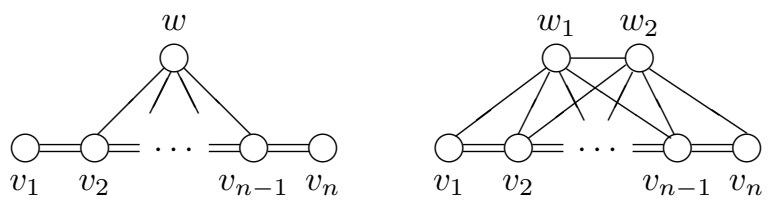

Fig. 4: Examples of non-SE graphs

A direct verification shows that neither a mountain nor a hill is an SE graph.

Definition 6 We refer to an induced path uvwx in a signed graph $G$ with $u v \in E_{\sigma}, v w \in E_{-\sigma}$, and $w x \in E_{\sigma}$, where $\sigma \in\{+,-\}$, as an alternating 4-path in $G$.

Then the characterization is given by the following theorem:

Theorem 1 Let $G$ be a signed graph. Then $G$ is an SE graph if and only if all of the following three conditions are satisfied:

(C1) Both $G_{+}$and $G_{-}$are chordal (i.e., having no induced cycle of length $\geq 4$ ).

(C2) For any alternating 4-path uvwx in $G$ with $u v \in E_{\sigma}$, we have either $u w \in E_{\sigma}$ and $u x \in E_{\sigma}$, or $u x \in E_{\sigma}$ and $v x \in E_{\sigma}$.

(C3) G contains no mountain and no hill as an induced subgraph.

An easy argument shows that the "only if" part of Theorem 1 holds, thus the nontrivial part of the theorem is to show that $G$ is an SE graph if the conditions (C1)-(C3) are satisfied. Moreover, since the conditions (C1)-(C3) are closed under taking an induced subgraph, the proof can be proceeded by induction on $|V|$. Note that the case $|V| \leq 3$ is trivial, since every signed graph with at most three vertices is an SE graph. Thus, by the properties mentioned in Section 2 it suffices to show that $S(G) \neq \emptyset$ if $G$ is connected, $E_{+} \neq \emptyset, E_{-} \neq \emptyset$, and $S(G \backslash\{v\}) \neq \emptyset$ for every vertex $v$ of $G$.

We explain an observation for the proof. For any graph $G$ and a subset $V^{\prime}$ of the vertex set $V$ of $G$, let $\left.G\right|_{V^{\prime}}$ denote the induced subgraph of $G$ with vertex set $V^{\prime}$. For a signed graph $G$, two subsets $V^{\prime}, V^{\prime \prime}$ of $V$ with $V^{\prime} \subset V^{\prime \prime}$, and $\sigma \in\{+,-\}$, let $W=\operatorname{cl}_{\sigma}\left(V^{\prime} ; V^{\prime \prime}\right)$ be the union of vertex sets of the connected components of $\left.G_{\sigma}\right|_{V^{\prime \prime}}$ that have nonempty intersection with $V^{\prime}$, and define

$$
\bar{W}=\overline{\operatorname{cl}}_{\sigma}\left(V^{\prime} ; V^{\prime \prime}\right)=\left\{v \in V^{\prime \prime} \mid N_{G_{-\sigma}}[v] \cap \operatorname{cl}_{\sigma}\left(V^{\prime} ; V^{\prime \prime}\right) \neq \emptyset\right\}
$$

(note that $W \subset \bar{W}$ ). Then it is shown that, if the condition (C2) is satisfied and every connected component of $\left.G_{\sigma}\right|_{W}$ contains at least two vertices, then any simplicial vertex of the "closure" $\left.G\right|_{\bar{W}}$ of $V^{\prime}$ 
relative to $V^{\prime \prime}$ is contained in the "interior set" $W$ of $\left.G\right|_{\bar{W}}$ and is also simplicial in $\left.G\right|_{V \text { ". Owing to this }}$ fact, by choosing an appropriate vertex $v$ of $G$, we can restrict possibilities of the simplicial vertices of the subgraph $G \backslash\{v\}$ (that exist by the induction hypothesis). This is a main tool of our proof, and a somewhat lengthy graph-theoretic argument enables us to find a desired simplicial vertex of the graph $G$. For the details, see a forthcoming full version of the article, or its preliminary version (Nuida (preprint 2007)).

We also present two lemmas for the proof that would be of independent interest:

Lemma 3 Let $G$ be a chordal graph and $V^{\prime} \subset V$ a clique of $G$ with $V^{\prime} \neq V$. Then there is a vertex $v \in V \backslash V^{\prime}$ such that $N_{G}[v]$ is a clique of $G$.

Lemma 4 Let $G$ be a connected $S E$ graph such that $E_{+} \neq \emptyset$ and $E_{-} \neq \emptyset$. Then $G$ has a signed-simplicial vertex $v$ such that $N_{G_{+}}[v] \neq\{v\}$ and $N_{G_{-}}[v] \neq\{v\}$.

In the last of this section, we state some special cases of our characterization:

Corollary 1 A signed graph $G$ with four vertices is $S E$ if and only if one of the following conditions is satisfied:

(FV1) Either $G_{+}$or $G_{-}$has a vertex of degree three.

(FV2) Both $G_{+}$and $G_{-}$are chordal, $G$ is not a mountain, and $G$ has no alternating 4-path.

Corollary 2 Let $G$ be a signed graph that is chordal (as a non-signed graph). Then $G$ is SE if and only if both conditions (C2) and (C3) are satisfied.

Corollary 3 Let $G$ be a signed graph with independence number $\alpha(G) \leq 2$ (i.e., every induced subgraph of $G$ with three vertices has an edge). Then $G$ is $S E$ if and only if the condition (C2) and the following two conditions are satisfied:

(I1) Both $G_{+}$and $G_{-}$have no cycle of length four or five that is an induced cycle in $G$.

(I2) G contains no hills with five or six vertices as an induced subgraph.

Corollary 4 Let $G$ be a signed graph that is a complete graph (as a non-signed graph). Then $G$ is $S E$ if and only if for each $\sigma \in\{+,-\}, G_{\sigma}$ contains, as an induced subgraph, neither a simple path with four vertices, nor a pair of two disjoint edges that are not joined by an edge in $G_{\sigma}$.

\section{Freeness Characterization of Multiplicities $2 k+m_{G}$}

Now we come back to the study of free multiarrangements mentioned in the Introduction. The full statement of our characterization of those free multiarrangements is the following:

Theorem 2 Let $\mathcal{A}=\mathcal{A}_{\ell}$ denote the braid arrangement in $V^{\ell+1}$ as in the Introduction, let $G$ be a signed graph with vertex set $V=\left\{v_{1}, \ldots, v_{\ell+1}\right\}$, and let $m_{G}$ be the map defined in (4). Let $k, n_{1}, \ldots, n_{\ell+1}$ be nonnegative integers. Let $m$ be a multiplicity on $\mathcal{A}$ defined by $m\left(H_{i j}\right)=2 k+n_{i}+n_{j}+m_{G}\left(H_{i j}\right)$ for each $H_{i j} \in \mathcal{A}$, and put $N=(\ell+1) k+\sum_{i=1}^{\ell+1} n_{i}$. Assume that one of the following three conditions is satisfied:

(a) $k>0$. 
(b) $E_{-}=\emptyset$.

(c) $E_{+}=\emptyset$ and $m\left(H_{i j}\right)>0$ for all $H_{i j} \in \mathcal{A}$.

Then $(\mathcal{A}, m)$ is free if and only if $G$ is an $S E$ graph. Moreover, if it is free, then the exponents of $(\mathcal{A}, m)$ are determined by

$$
\exp (\mathcal{A}, m)=\left(0, N+\widetilde{\operatorname{deg}}_{2}, \ldots, N+\widetilde{\operatorname{deg}}_{\ell+1}\right)
$$

where $\widetilde{\operatorname{deg}_{i}}$ is the quantity associated to $G$ defined in $(6)$.

Note that, in the case that $E_{-}=\emptyset$ and $k=n_{1}=\cdots=n_{\ell+1}=0$, the multiarrangement in Theorem 2 coincides with a graphic arrangement mentioned in the Introduction. Thus Theorem 2 is a generalization of Stanley's aforementioned characterization of free graphic arrangement (see Stanley (1972)).

We explain an outline of the proof of Theorem 2. First, for the "if" part, suppose that $G$ is an SE graph with an SEO $\nu$. By an appropriate permutation of coordinates, we assume without loss of generality that $\nu\left(v_{i}\right)=i$ for every $i$. We proceed the proof by induction on $\ell$, and the case $\ell \leq 2$ follows from the result of Wakamiko (2007). For the case $\ell>2$, Proposition 1 implies that $G$ has a complete SE filtration $G_{0}^{\prime}, G_{1}^{\prime}, \ldots, G_{r}^{\prime}=G$ corresponding to the SEO $\nu$. We show by induction on $i$ that the multiarrangement $\left(\mathcal{A}^{(i)}, m^{(i)}\right)$ corresponding to each SE graph $G_{i}^{\prime}$ is free. For the step from $G_{i-1}^{\prime}$ to $G_{i}^{\prime}$, let $v_{j} v_{k}$ denote the edge added to $G_{i-1}^{\prime}$ in this step, where $j<k$. Let $\mathcal{A}^{\prime}=\left\{H \cap H_{j k} \mid H \in \mathcal{A}^{(i)} \backslash\left\{H_{j k}\right\}\right\}$, which is an arrangement in an $\ell$-dimensional space, and let $\left(\mathcal{A}^{\prime}, m^{\prime}\right)$ be a certain special multiarrangement (called the Euler restriction of $\left(\mathcal{A}^{(i)}, m^{(i)}\right)$ to $H_{j k}$ ) obtained by a result of Abe-Terao-Wakefield (2008). Then it follows from results of Abe-Terao-Wakefield (2008) that $\left(\mathcal{A}^{\prime}, m^{\prime}\right)$ is a multiarrangement of the form in Theorem 2 corresponding to an induced subgraph of $G$ and the restriction of $\nu$ to this subgraph, therefore the first induction hypothesis implies that $\left(\mathcal{A}^{\prime}, m^{\prime}\right)$ is free. Now owing to Addition-Deletion Theorem (Theorem 0.8 of Abe-Terao-Wakefield (2008)), freeness of $\left(\mathcal{A}^{(i-1)}, m^{(i-1)}\right)$ implies freeness of $\left(\mathcal{A}^{(i)}, m^{(i)}\right)$, therefore the claim follows from the second induction hypothesis. Thus the "if" part is proved. Moreover, the description of the exponents is also obtained by the same argument in parallel. Note that existence of complete SE filtrations plays a key role in this proof, but the characterization of SE graphs has not yet appeared.

On the other hand, for the "only if" part, we show that $(\mathcal{A}, m)$ is not free if $G$ is not an SE graph. Then by the characterization of SE graphs (Theorem 11), one of the three conditions (C1)-(C3) is not satisfied, namely we are in one of the following situations:

- Either $G_{+}$or $G_{-}$has an induced cycle of length $\geq 4$.

- $G$ contains an alternating 4-path $u v w x$ with $u v \in E_{\sigma}$ such that either $u x \notin E_{\sigma}$, or $u w \notin E_{\sigma}$ and $v x \notin E_{\sigma}$.

- $G$ contains a mountain or a hill as an induced subgraph.

Owing to Lemma 3.8 in Abe (2006), it suffices to show that the multiarrangement corresponding to the subgraph of $G$ specified in the above conditions is not free. This is done by a case-by-case argument based on Addition-Deletion Theorem and other preceding results of Abe-Terao-Wakefield (2008), Abe-TeraoWakefield (2007), and Wakamiko (2007). Thus the "only if" part is proved. For the details, see the full version of this article (Abe-Nuida-Numata (2009)). 
We would like to summarize here again that the complete SE filtration plays a key role in the proof of the "if" part of Theorem 2, while the proof of the "only if" part requires our characterization of SE graphs given in Theorem 1 .

In the last of this section, we give a remark on characteristic polynomials of the above multiarrangements that is an easy consequence of Theorem 2 Characteristic polynomials $\chi(\mathcal{A}, m, t)$ of multiarrangements $(\mathcal{A}, m)$ are defined by Abe-Terao-Wakefield (2007), and in that article a factorization theorem of $\chi(\mathcal{A}, m, t)$ is proved. It is difficult to compute the polynomial $\chi(\mathcal{A}, m, t)$ in general. However, if $(\mathcal{A}, m)$ is a free multiarrangement, then the computation becomes easy owing to the factorization theorem. Thus by Theorem 2, we obtain the following result on characteristic polynomials of the above multiarrangements:

Corollary 5 Let $(\mathcal{A}, m)$ be the same multiarrangement as in Theorem 2 corresponding to a signed graph G. Let $\widetilde{m}$ be another multiplicity on $\mathcal{A}$ such that $\widetilde{m}\left(H_{i j}\right)=2 k+n_{i}+n_{j}-m_{G}\left(H_{i j}\right)$ for each $H_{i j} \in \mathcal{A}$. Then, in the case $k>0,(\mathcal{A}, m)$ is free if and only if $(\mathcal{A}, \widetilde{m})$ is free. Moreover, if $G$ is an $S E$ graph, then we have

$$
\chi(\mathcal{A}, m, t)=t \prod_{i=2}^{\ell+1}\left(t-N-\widetilde{\operatorname{deg}_{i}}\right)
$$

and

$$
\chi(\mathcal{A}, \widetilde{m}, t)=t \prod_{i=2}^{\ell+1}\left(t-N+\widetilde{\operatorname{deg}_{i}}\right) .
$$

\section{Conjecture of Athanasiadis}

In this section, we explain a relation of our result with a conjecture of Athanasiadis (see Athanasiadis (2000)) on graphic characterization of free arrangements in another class. Here we consider a non-central affine arrangement in $V^{\ell+1}$ that consists of affine hyperplanes defined by $x_{i}-x_{j}=h(1 \leq i<j \leq \ell+1)$, where $h \in \mathbb{Z},-k-\varepsilon(i, j) \leq h \leq k+\varepsilon(j, i), k \in \mathbb{Z}_{\geq 0}$ is a constant, and $\varepsilon(i, j) \in\{0,1\}$. Such an arrangement is called a deformation of the Coxeter arrangement and was first systematically investigated by Stanley (1996). These arrangements have been extensively studied by several persons such as Athanasiadis (1996, 1998, 2000), Edelman-Reiner (1996), Postnikov-Stanley (2000), and Yoshinaga (2004). In particular, Athanasiadis (Athanasiadis (1996)) introduced a description of the above arrangement in terms of a directed graph $G=(V, E)$ with vertex set $V=\left\{v_{1}, \ldots, v_{\ell+1}\right\}$. For such a graph $G$, define $\varepsilon(i, j)=1$ if $\left(v_{i}, v_{j}\right) \in E$ and $\varepsilon(i, j)=0$ if $\left(v_{i}, v_{j}\right) \notin E$, where $\left(v_{i}, v_{j}\right)$ denotes an arrow from $v_{i}$ to $v_{j}$. Note that every affine arrangement of the above form is parameterized in this manner. Let $\mathcal{A}_{G}$ denote the arrangement corresponding to $G$. Then Athanasiadis (Athanasiadis (1996)) gives a splitting formula of the characteristic polynomial of $\mathcal{A}_{G}$ in the case that $G$ satisfies the following two conditions:

(A1) For every triple $v_{h}, v_{i}, v_{j}$ of vertices of $G$ with $i<h$ and $j<h,\left(v_{i}, v_{j}\right) \in E$ implies either $\left(v_{i}, v_{h}\right) \in E$ or $\left(v_{h}, v_{j}\right) \in E$.

(A2) For every triple $v_{h}, v_{i}, v_{j}$ of vertices of $G$ with $i<h$ and $j<h$, we have $\left(v_{i}, v_{j}\right) \in E$ if $\left(v_{i}, v_{h}\right) \in$ $E$ and $\left(v_{h}, v_{j}\right) \in E$.

Moreover, he also gave a conjecture (Conjecture 6.6 in Athanasiadis (2000)) that in the case $k=0$, the conditions (A1) and (A2) would be necessary and sufficient for the "coning" $c \mathcal{A}_{G}$ of $\mathcal{A}_{G}$ to be a free 
arrangement. We mention that one direction of Athanasiadis's Conjecture is proved in a more general setting by applying our results in the previous sections. Namely, we have the following result:

Theorem 3 In the above setting, where we do not assume $k=0$, the coning $c \mathcal{A}_{G}$ of $\mathcal{A}_{G}$ is free if $G$ satisfies the conditions (A1) and (A2).

We give an outline of the proof of Theorem 3 . Let $H_{\infty}$ be the infinity hyperplane of the coning $c \mathcal{A}_{G}$ of $\mathcal{A}_{G}$. Let $\left(\mathcal{A}^{\prime \prime}, m_{H_{\infty}}\right)$ denote the Ziegler restriction of $c \mathcal{A}_{G}$ (see Ziegler $(1989)$ ) defined by $\mathcal{A}^{\prime \prime}=$ $\left\{H \cap H_{\infty} \mid H_{\infty} \neq H \in c \mathcal{A}_{G}\right\}$ and $m_{H_{\infty}}(X)=\left|\left\{H \in c \mathcal{A}_{G} \backslash\left\{H_{\infty}\right\} \mid H \cap H_{\infty}=X\right\}\right|$ for each $X \in \mathcal{A}^{\prime \prime}$. Then it is shown that $\left(\mathcal{A}^{\prime \prime}, m_{H_{\infty}}\right)$ is of the form in Theorem 2 corresponding to a signed graph $G^{\prime}$, and the conditions (A1) and (A2) for $G$ imply that $G^{\prime}$ is an SE graph. Thus $\left(\mathcal{A}^{\prime \prime}, m_{H_{\infty}}\right)$ is free by Theorem 2. Now owing to Theorem 2.2 of Yoshinaga (2004), an argument based on induction on $\ell$ implies that $c \mathcal{A}_{G}$ is free. Thus Theorem 3 is proved. For the details, see the full version of this article (Abe-Nuida-Numata (2009)).

\section{Acknowledgements}

The authors would like to thank Professor Masahiko Yoshinaga, and Professor Max Wakefield, for their precious comments and suggestions. Moreover, the authors would like to thank the anonymous referees for their significant comments.

\section{References}

T. Abe. The freeness of $A_{2}$ and $B_{2}$-type arrangements and lattice cohomologies. In: Recent Topics on Real and Complex Singularities. RIMS Kôkyûroku 1501, Kyoto University, 2006, pp.31-46.

T. Abe. Free and non-free multiplicity on the deleted $A_{3}$ arrangement. Proc. Japan Acad. Ser. A 83 (2007) 99-103.

T. Abe. A generalized logarithmic module and duality of Coxeter multiarrangements. arXiv:0807.2552v1 (math.AC), 2008.

T. Abe, K. Nuida, and Y. Numata. Signed-eliminable graphs and free multiplicities on the braid arrangement. J. London Math. Soc. (2009); doi: 10.1112/jlms/jdp019

T. Abe, H. Terao, and M. Wakefield. The characteristic polynomial of a multiarrangement. Adv. in Math. 215 (2007) 825-838.

T. Abe, H. Terao, and M. Wakefield. The Euler multiplicity and addition-deletion theorems for multiarrangements. J. London Math. Soc. 77(2) (2008) 335-348.

T. Abe and M. Yoshinaga. Coxeter multiarrangements with quasi-constant multiplicities. arXiv:0708.3228.

C. A. Athanasiadis. Characteristic polynomials of subspace arrangements and finite fields. Adv. in Math. 122 (1996) 193-233.

C. A. Athanasiadis. On free deformations of the braid arrangement. European J. Combin. 19 (1998) $7-18$. 
C. A. Athanasiadis. Deformations of Coxeter hyperplane arrangements and their characteristic polynomials. In: Arrangements - Tokyo 1998. Advanced Studies in Pure Mathematics 27, Kinokuniya, Tokyo, 2000, pp. 1-26.

R. Diestel. Graph Theory, third ed. Graduate Texts in Mathematics 173, Springer-Verlag, New York, 2006.

P. H. Edelman and V. Reiner. Free arrangements and rhombic tilings. Discrete Comp. Geom. 15 (1996) 307-340.

D. R. Fulkerson and O. A. Gross. Incidence matrices and interval graphs. Pacific J. Math. 15(3) (1965) 835-855.

K. Nuida. A characterization of signed graphs with generalized perfect elimination orderings. arXiv:0712.4118v3 (math.CO), 2007.

P. Orlik and H. Terao. Arrangements of hyperplanes. Grundlehren der Mathematischen Wissenschaften 300, Splinger-Verlag, Berlin, 1992.

A. Postnikov and R. P. Stanley. Deformations of Coxeter hyperplane arrangements. J. Combin. Theory Ser. A 91 (2000) 544-597.

D. J. Rose. Triangulated graphs and the elimination process. J. Math. Anal. Appl. 32 (1970) 597-609.

K. Saito. On the uniformization of complements of discriminant loci. In: Conference Notes. Amer. Math. Soc. Summer Institute, Williamstown, 1975.

K. Saito. Theory of logarithmic differential forms and logarithmic vector fields. J. Fac. Sci. Univ. Tokyo Sect. IA Math. 27 (1980) 265-291.

L. Solomon and H. Terao. The double Coxeter arrangements. Comment. Math. Helv. 73 (1998) 237-258.

R. P. Stanley. Supersolvable lattices. Algebra Universalis 2 (1972) 197-217.

R. P. Stanley. Hyperplane arrangements, interval orders and trees. Proc. Natl. Acad. Sci. 93 (1996) $2620-2625$.

H. Terao. Multiderivations of Coxeter arrangements. Invent. Math. 148 (2002) 659-674.

A. Wakamiko. On the exponents of 2-multiarrangements. Tokyo J. Math. 30 (2007) 99-116.

M. Yoshinaga. The primitive derivation and freeness of multi-Coxeter arrangements. Proc. Japan Acad. Ser. A 78 (2002) 116-119.

M. Yoshinaga. Characterization of a free arrangement and conjecture of Edelman and Reiner. Invent. Math. 157 (2004) 449-454.

M. Yoshinaga. On the extendability of free multiarrangements. arXiv:0710.5044.

G. M. Ziegler. Multiarrangements of hyperplanes and their freeness. In: Singularities (Iowa City, IA, 1986), 345-359, Contemp. Math. 90, Amer. Math. Soc., Providence, RI, 1989. 